\title{
Resonant Bending Fatigue Tests on Thin Films
}

\author{
Kwangsik Kwak, Masaaki Otsu and Kazuki Takashima* \\ Department of Materials Science and Engineering, Kumamoto University, \\ 2-39-1 Kurokami, Kumamoto, Japan
}

(Received July 9, 2009; accepted August 24, 2009)

Key words: structural thin films, fatigue, fatigue strength, S-N curve, Ni-P amorphous, Au, single-crystal silicon

The fatigue properties of thin-film materials are extremely important in the design of durable and reliable micro-electromechanical systems (MEMS). However, it is rather difficult to apply the conventional fatigue testing method for bulk materials to thin films as the specimen size is extremely small. Therefore, a fatigue testing method suitable for thin-film materials is required. We have developed a fatigue testing method that uses the resonance of a cantilever-type specimen prepared from thin films. Cantilever beam specimens with dimensions of $3(L) \times 1(W) \times 0.01(t) \mathrm{mm}^{3}$ were prepared from Ni-P amorphous alloy thin films and gold foils. In addition, cantilever beam specimens with dimensions of $3(L) \times 0.3(W) \times 0.005(t) \mathrm{mm}^{3}$ were prepared from single-crystal silicon thin films. These specimens were fixed to a holder that was connected to an audio speaker (actuator) and were resonated in the bending mode. The Young's moduli measured from the resonant frequencies of Ni-P and gold foil were 116 and $72 \mathrm{GPa}$, respectively. These values were comparable to those measured by other techniques, indicating that the specimens resonated in a theoretically predictable manner and that our method is valid. Resonant fatigue tests were carried out for these specimens by changing the amplitude range of resonance, and S-N curves were successfully obtained for Ni-P amorphous alloy thin films, gold foils, and single-crystal silicon thin films.

\section{Introduction}

In micro-electromechanical systems (MEMS), micromechanical components are used in the construction of moving parts, including microbeams and membranes. These microcomponents are prepared from thin films deposited onto a substrate using suitable micromachining processes. The evaluation of mechanical properties including the elastic modulus, tensile strength, fracture toughness, and fatigue life of such structural thin films is, therefore, necessary to enable the design of reliable MEMS devices. In particular, knowledge of the fatigue properties of thin-film materials is vital for the design of durable MEMS devices. Fatigue tests on thin-film materials have been carried out with proportionally miniaturized dog-born type or cantilever bending specimens using the same testing method performed for ordinary-sized bulk materials. ${ }^{(1)}$ In these testing

*Corresponding author: e-mail: takashik@gpo.kumamoto-u.ac.jp 
methods, the cyclic loading frequency is usually up to $10 \mathrm{~Hz}$, and it takes much time to obtain a sufficient fatigue strength after $10^{9}-10^{10}$ cycles, which are required for designing MEMS devices. Therefore, on-chip resonating fatigue testing methods at a frequency of several tens of $\mathrm{kHz}$ have been developed to reduce testing time in the high-frequency fatigue region. ${ }^{(2-5)}$ In this testing structure, however, a comb-driven actuator and a specimen are prepared concurrently by a photolithography process. This indicates that this technique is applicable to only thin films on substrates. For a cantilever specimen, if the length is much larger than the thickness, bending resonance will easily occur by applying vibration. During the resonant vibration, cyclic stress is applied at the fixed end of the cantilever. This indicates that fatigue tests can be performed by bending resonance using simple cantilever-type specimens. In addition, cantilever-type specimens can be prepared easily for both free-standing thin films and thin films on substrates even if photolithography processes are used. In this investigation, a fatigue testing method for thin films by bending resonance has been developed and fatigue tests on Ni-P amorphous alloy thin films, gold foils, and single-crystal silicon films have been performed.

\section{Experimental Procedure}

\subsection{Materials and specimens}

The materials used were Ni-P amorphous alloy films, gold foils, and single-crystal silicon (SCS) thin films. A Ni-11.5 mass\% P amorphous alloy film was produced by electroless plating onto an Al-4.5 mass $\% \mathrm{Mg}$ substrate. This produced an amorphous layer of $12 \mu \mathrm{m}$ thickness on a $0.79-\mathrm{mm}$-thick substrate. ${ }^{(1,6)}$ The specimen was cut from the Ni-P/Al-Mg into a rectangular parallelepiped shape, prior to the removal of the substrate by dissolution in a $\mathrm{NaOH}$ aqueous solution. The length $(L)$, width $(W)$ and thickness $(t)$ of specimens were $\approx 10 \mathrm{~mm}, 1.5 \mathrm{~mm}$ and $12 \mu \mathrm{m}$, respectively, as shown in Fig. 1(a). Gold foil specimens were prepared from cold-rolled gold tape $(99.99 \%$ purity) with a width of $1.4 \mathrm{~mm}$ and a thickness of $10 \mu \mathrm{m}$. This tape was cut to $10 \mathrm{~mm}$ in

(a)

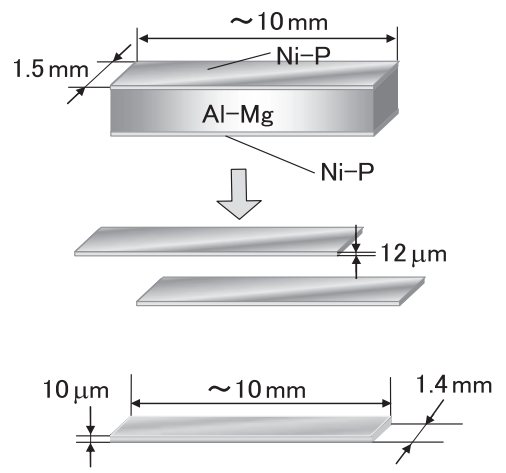

Fig. 1. Geometries and preparation of (a) Ni-P amorphous alloy thin-film specimen and (b) gold foil specimen. 
length, as shown in Fig. 1(b). The average grain size of the gold foil was $0.73 \mu \mathrm{m}$. SCS specimens were fabricated from the top layer of silicon-on-insulator (SOI) wafers using a photolithography process. The cantilever beams were $3 \mathrm{~mm}$ in length, $300 \mu \mathrm{m}$ in width, and $5 \mu \mathrm{m}$ in thickness. In addition, a weight was put at the free end of the specimen, as shown in Fig. 2. The direction of the cantilever beam was [110]. For SCS specimens, a through thickness notch was made at the center of the specimen by focused ion beam (FIB) machining, as shown in Fig. 3. The notch length was $100 \mu \mathrm{m}$, which was located $50 \mu \mathrm{m}$ from the fixed end of the specimen. The width of the notch was $0.5 \mu \mathrm{m}$.

\subsection{Fatigue testing machine}

A fatigue testing method that uses the resonance of a cantilever-type specimen prepared from thin films was developed in this investigation. Figure 4 shows a block diagram of the fatigue testing machine. The testing machine consists of an audio speaker (FORSTER FF-77EG $8 \Omega / 5 \mathrm{~W}$ ), a laser displacement meter (KEYENCE LK-G30), a function generator (NF WF1973), and an audio amplifier. An audio speaker was used as an actuator, which causes the cantilever-type specimen to vibrate. Ni-P amorphous alloy

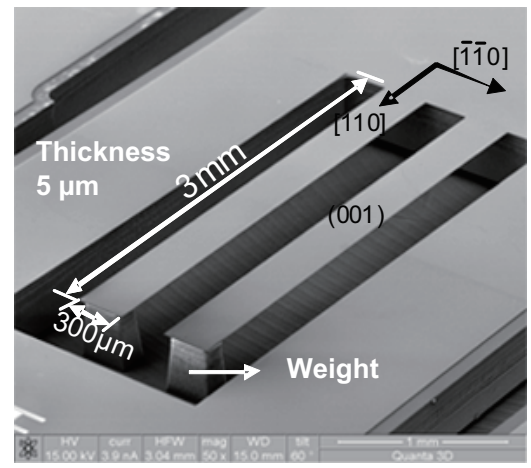

Fig. 2. Scanning electron micrograph of microcantilever specimen prepared from single-crystal silicon thin film.

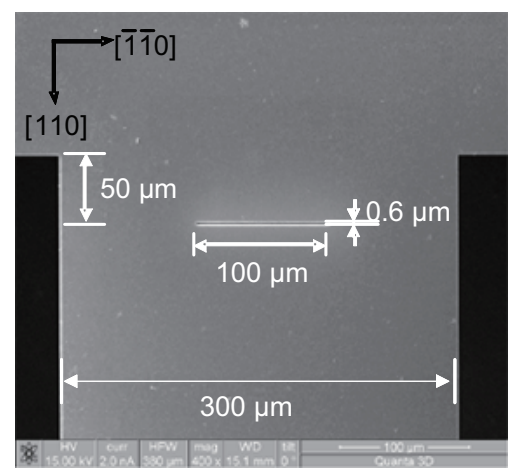

Fig. 3. Notch geometry of single-crystal silicon specimen. 


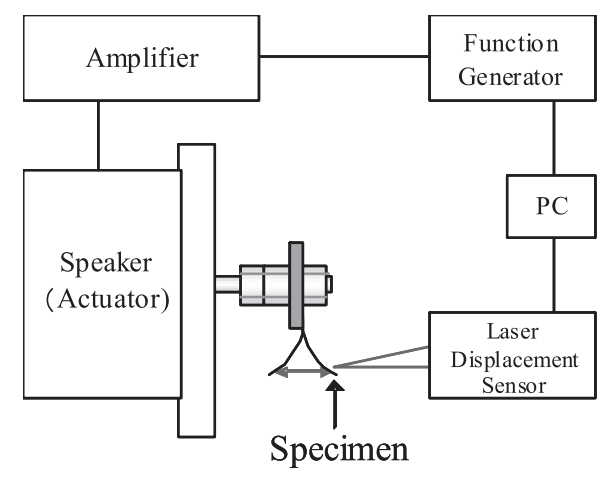

Fig. 4. Block diagram of resonant fatigue testing equipment.

and $\mathrm{Au}$ foil specimens were fixed on a fixture set on the speaker cone. The cantilever specimen of SCS silicon was set in a specimen holder and the holder was placed on the top of the speaker cone, as shown in Fig. 4. A sine wave generated by the function generator was used as the driving signal of the speaker. The output signal of the function generator was amplified by the audio amplifier. The displacement amplitude of the specimen was measured by the laser displacement meter. The spot size of the laser is $30 \mu \mathrm{m}$, which may cause measurement error, however, it is deduced to be only $1.5 \%$ in this testing. This error is small enough when considering the scatter of the fatigue life of the specimen, so the displacement error can be neglected. The resonance of the specimen was also monitored by a CCD camera that was set near the specimen holder. Fatigue tests were carried out at room temperature in air, and the humidity was kept at $50-60 \%$ relative humidity $(\mathrm{RH})$ during the tests. The resonant frequency may change during the fatigue test, but as mentioned in the next section, the fatigue fracture occurred abruptly for all specimens, so the displacement of vibration was kept constant throughout the fatigue test.

\section{Results and Discussion}

\subsection{Resonant behavior}

Prior to fatigue testing, the resonant condition of the specimen was examined. Figure 5 shows the relationship between the displacement amplitude range and frequency of the Ni-P amorphous alloy thin-film specimen. The resonance of the specimen is observed at a frequency of $180 \mathrm{~Hz}$. This type of resonance was also confirmed for the gold foil and SCS specimens; the resonant frequency of the gold foil specimen was 163 $\mathrm{Hz}$ and that of the SCS specimen was $175 \mathrm{~Hz}$. In order to check the validity of this testing method, Young's moduli of the specimens were calculated from the resonant frequencies. The fundamental resonance frequency of a cantilever beam is given by

$$
f_{\mathrm{c}}=0.16154 \frac{h}{L^{2}} \sqrt{\frac{E_{\mathrm{e}}}{\rho}}
$$




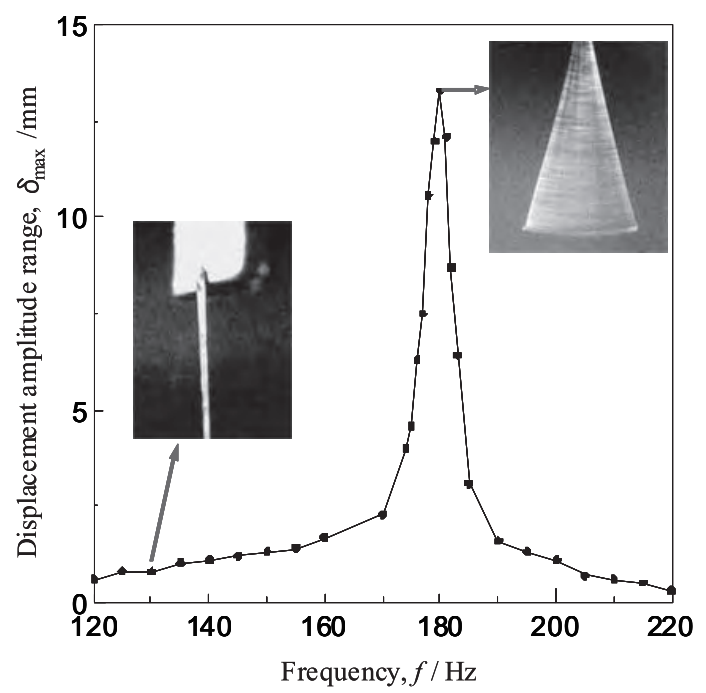

Fig. 5. Relationship between displacement amplitude range and frequency for Ni-P amorphous alloy thin-film specimen.

where $L$ and $h$ are the length and thickness of the cantilever beam, respectively, $E_{\mathrm{e}}$ is the effective Young's modulus, and $\rho$ is the density of the material. ${ }^{(7)}$ The effective modulus, $E_{\mathrm{e}}$, is replaced by $E /\left(1-v^{2}\right)$, where $v$ is Poisson's ratio and $E$ is Young's modulus, if the beam width $b$ is larger than the beam thickness $h(b \geq 5 h) .{ }^{(7)}$ From the resonant frequency, the average Young's modulus of the Ni-P amorphous alloy thin-film specimen was calculated to be $116 \mathrm{GPa}$, which is consistent with that of the Ni-P amorphous alloy thin film measured by other techniques $(110 \mathrm{GPa}){ }^{(8)}$ The average Young's modulus of the gold foil was $71.6 \mathrm{GPa}$, which is also consistent with that of polycrystalline gold (68.4 $\mathrm{GPa}){ }^{(7)}$ These results show that the resonance vibration of the cantilever beam occurred in the theoretically predicted manner, and this technique can be used to conduct a fatigue test on thin-film specimens. Resonant vibration was also observed for the SCS cantilever specimen, but Young's modulus could not be determined since the weight was attached to the free end of the beam and the notch was introduced in the SCS specimens, as shown in Fig. 2.

\subsection{Stress-life curves}

Fatigue tests were performed by changing the displacement amplitude range. The maximum stress during bending resonance was calculated using eq. (2):(9)

$$
\sigma_{\max }=\frac{2 h \delta E}{L^{2}}
$$

where $\sigma_{\max }$ and $\delta$ are maximum bending stress and maximum displacement, respectively. 
Figure 6 shows the S-N plots for the Ni-P amorphous alloy thin films. Because the maximum stress over the fatigue cycle was lower than the static strength of the Ni-P amorphous alloy (approximately $1 \mathrm{GPa}$ ), only a few specimens suffered from fatigue fracture, and thus fatigue strength could not be determined. Figure 7 shows the S-N plots for gold foils. The fatigue strength was $180 \mathrm{MPa}$ after $10^{6}$ cycles. The tensile strength $\left(\sigma_{\mathrm{B}}\right)$ of a gold film with similarly sized grains has been reported to be approximately 350 $\mathrm{MPa},{ }^{(10)}$ and the fatigue strength of this gold foil corresponds to $0.51 \sigma_{\mathrm{B}}$. This value is slightly high compared with the fatigue strength of ordinary bulk metals $\left(0.25-0.3 \sigma_{\mathrm{B}}\right)$. A similar trend was also observed for the bending fatigue of an electroless deposited Ni-P amorphous alloy, ${ }^{(6)}$ but no details were given.

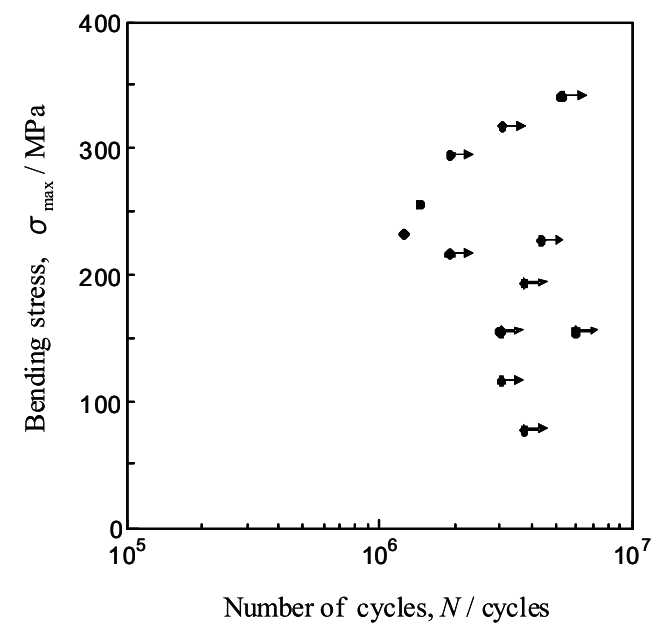

Fig. 6. S-N plots for Ni-P amorphous alloy thin-film specimens.

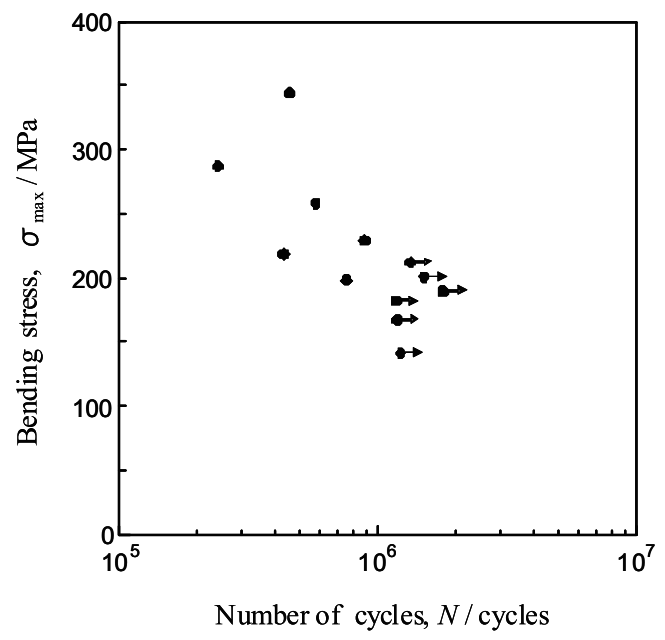

Fig. 7. S-N plots for Au foil specimens. 
Fatigue fracture does not occur for SCS specimens without a notch in this testing method, since the applied stress at the fixed end is too small. The resonant fatigue test was then performed for notched specimens, as shown in Fig. 3. Figure 8 shows $\mathrm{S}-\mathrm{N}$ plots for notched SCS specimens. The vertical axis in Fig. 8 indicates the ratio of the applied displacement to the average fracture displacement at the instantaneous occurrence of fracture, which corresponds to the ratio of the applied stress to the static strength of the specimen. The fatigue lives are randomly distributed, and the fatigue strength in the high-cycle region $\left(10^{8}\right.$ cycles $)$ did not decrease compared with that in the low-cycle region. A similar behavior is often observed for the fatigue of silicon films. ${ }^{(4-6)}$ One interesting feature of the plot is that fatigue does not occur in the range between $10^{4}$ and $10^{6}$ cycles under the stress conditions tested in this investigation, but the reason for this is not clarified herein.

\subsection{Fatigue-fractured surface}

Figure 9 shows a scanning electron micrograph of the Ni-P amorphous alloy thinfilm specimen after a fatigue fracture with a fatigue life of $1.5 \times 10^{6}$ cycles. Striations are clearly observed on the fracture surface at about $0.5 \mu \mathrm{m}$ intervals. This type of striation is also observed on the fatigue surface of Ni-P thin films. ${ }^{(2)}$ This indicates that the specimen was actually fractured by cyclic loading.

For gold foil specimens, the vibration displacement started to decrease from approximately $10^{4}$ cycles before the final fracture, and the final fracture occurred abruptly. Figure 10 shows a fatigue surface of the gold foil specimen after $9.0 \times 10^{5}$ cycles. Distinct asperities are observed near the crack surface, and small grains are also seen in the deformed area. The grain size of the Au foil is approximately $0.73 \mu \mathrm{m}$ and this size is comparable to that of the asperities of this material. This suggests that the crack has propagated along the grain boundary. The Au foil used in this study was fabricated by cold rolling, and many dislocations were present in the grain. This suggests

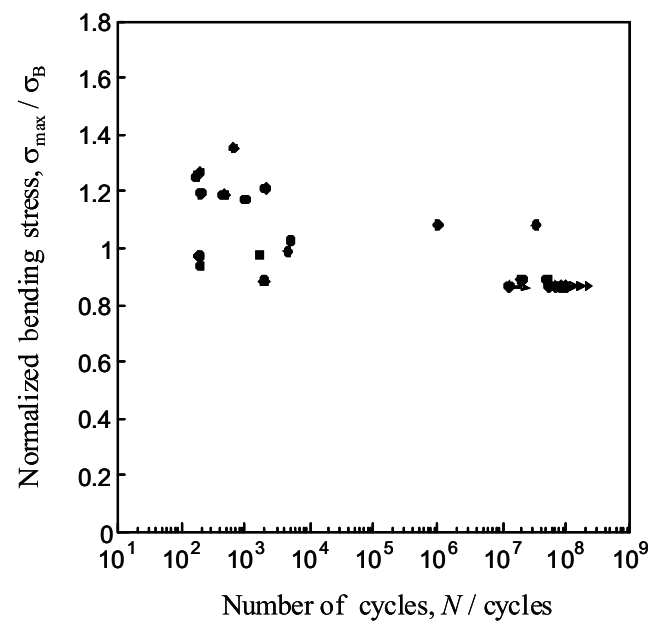

Fig. 8. S-N plots for SCS specimens. 


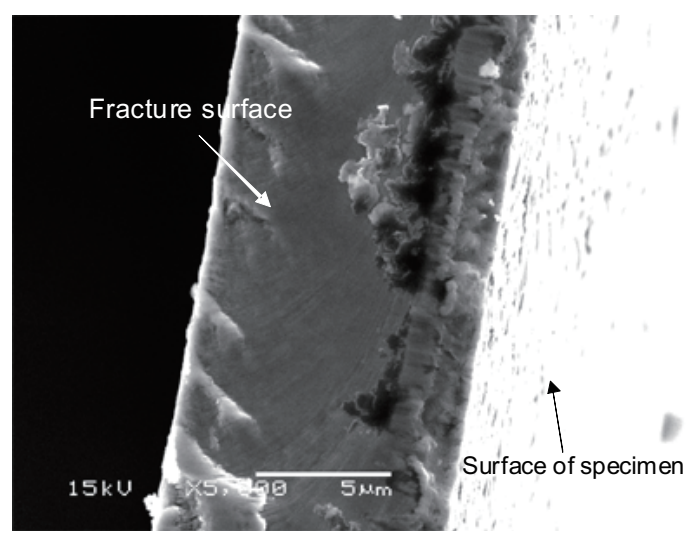

Fig. 9. Scanning electron micrograph of fracture surface of Ni-P thin film after $1.5 \times 10^{6}$ cycles.

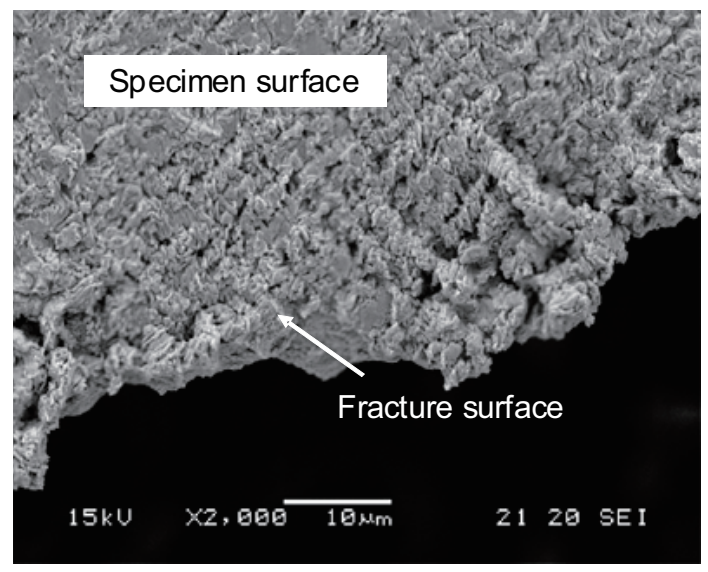

Fig. 10. Scanning electron micrograph of fracture surface of Au foil after $1 \times 10^{6}$ cycles.

that further plastic deformation of each grain is difficult. Therefore, it was deduced that cracking occurs at the grain boundary, and that the fatigue crack grows between the grain boundaries.

Figure 11 shows a fracture surface of the SCS thin-film specimen. Figure 11(a) shows a fatigue-fractured surface of the specimen after $5.0 \times 10^{7}$ cycles. The fracture surface is very flat and shows a cleavagelike feature, but some steplike regions are also found on the specimen surface. The crack surface is parallel to the notch plane, which is the (110) plane. Figure 11(b) shows the fatigue-fractured surface of the specimen after 1.9 $\times 10^{2}$ cycles (relatively short life). Some flaws are seen on the fracture surface near the notch. It is very likely that these defects were introduced into the SCS thin films during processing. These defects shorten the fatigue life of the SCS specimen. This suggests that fatigue life is dependent on the internal (or surface) defects of materials. 

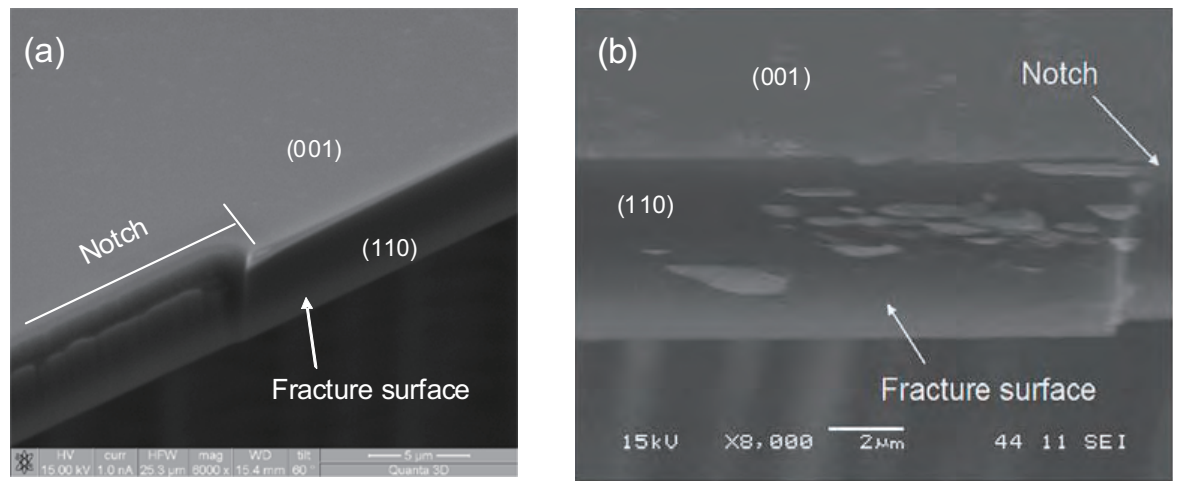

Fig. 11. Scanning electron micrographs of fracture surface of SCS. (a) Fatigue life: $5.0 \times 10^{7}$ cycles and (b) fatigue life: $1.9 \times 10^{2}$ cycles.

\section{Conclusions}

Bending resonance fatigue tests have been performed for microsized cantilever beamtype specimens prepared from Ni-P amorphous alloy thin films, gold foils, and singlecrystal silicon thin films. Resonant fatigue tests were carried out successfully for these specimens, and S-N curves were obtained. This testing method is useful for measuring the fatigue life of thin-film materials.

\section{References}

1 K. Takashima and Y. Higo: Fatigue Fract. Eng. M. 28 (2005) 703.

2 J.-S. Bae, C.-S. Oh, K.-S. Park, S.-K. Kim and H.-J. Lee: Eng. Fract. Mech. 75 (2008) 4958.

3 C. L. Muhlstein, E.A. Stach and R.O. Ritchie: Acta Mater. 50 (2002) 3579.

4 H. K. Liu, B. J. Lee and P. P Liu: Sens. Actuators, A 140 (2007) 257.

5 C. L. Muhlstein, S. B. Brown and R. O. Ritchie: J. Microelectromech. Syst. 10 (2001) 593.

6 K. Takashima, Y. Higo, S. Sugiura and M. Shimojo: Mater. Trans. 42 (2001) 68.

7 C.-W. Baek, Y.-K. Kim, Y. Ahn and Y.-H. Kim: Sens. Actuators, A 117 (2005) 17.

8 G. Li, Y. P. Gao and R. P. Liu: J. Non-Cryst. Solids 353 (2007) 4199.

9 S. Nakano, R. Maeda and K. Yamanaka: Jpn. J. Appl. Phys. 36 (1997) 3265.

10 R. D. Emery and G. L. Povirk: Acta Mater. 51 (2003) 2067. 